\title{
Genome and RNA sequencing in patients with methylmalonic aciduria of unknown cause
}

\author{
Lina Sobhi Abdrabo, MSc, MD ${ }^{1}$, David Watkins, PhD ${ }^{1,2}$, Sophie Ran Wang, PhD ${ }^{1}$, \\ Joël Lafond-Lapalme, $\mathrm{MSc}^{1}$, Jean-Baptiste Riviere, $\mathrm{PhD}^{1,2}$ and David S. Rosenblatt, MD ${ }^{1,2,3}$
}

Purpose: Our laboratory has classified patients with methylmalonic aciduria using somatic cell studies for over four decades. We have accumulated 127 fibroblast lines from patients with persistent elevated methylmalonic acid (MMA) levels in which no genetic cause could be identified. Cultured fibroblasts from 26 of these patients had low $\left[{ }^{14} \mathrm{C}\right]$ propionate incorporation into macromolecules, possibly reflecting decreased methylmalonyl-CoA mutase function.

Methods: Genome sequencing (GS), copy-number variation $(\mathrm{CNV})$ analysis, and RNA sequencing were performed on genomic DNA and complementary DNA (cDNA) from these 26 patients.

Results: No patient had two pathogenic variants in any gene associated with cobalamin metabolism. Nine patients had heterozygous variants of unknown significance previously identified by a next-generation sequencing (NGS) panel targeting cobalamin metabolic genes. Three patients had pathogenic changes in genes not associated with cobalamin metabolism (PCCA, EPCAM, and a $17 \mathrm{q} 12$ duplication) that explain parts of their phenotypes other than elevated MMA.

Conclusion: Genome and RNA sequencing did not detect any additional putative causal genetic defects in known cobalamin genes following somatic cell studies and the use of a targeted NGS panel. They did detect pathogenic variants in other genes in three patients that explained some aspects of their clinical presentation.

Genetics in Medicine (2020) 22:432-436; https://doi.org/10.1038/ s41436-019-0640-9

Keywords: methylmalonic aciduria; genome sequencing; RNA sequencing; vitamin $\mathrm{B}_{12}$; cobalamin

\section{INTRODUCTION}

Genome sequencing (GS) and transcriptome studies have become important diagnostic tools. ${ }^{1,2}$ These technologies identify a vastly larger number of genetic variants than exome sequencing (ES), but they come with greater costs, and an additional need for high-performance computing for data analysis and storage.

Inborn errors of cobalamin $(\mathrm{Cbl})$ metabolism give rise to an array of clinical disorders characterized by elevations of methylmalonic acid (MMA) and/or homocysteine (Hcy) in blood and/or urine, and hematological, neurological, and other manifestations. ${ }^{3}$ Adenosylcobalamin (AdoCbl) is required as a cofactor for methylmalonyl-CoA mutase $(\mathrm{MCM})$ in the production of succinyl-CoA from methylmalonyl-CoA. In the Vitamin $B_{12}$ Laboratory at McGill University, somatic cell techniques have been established for the classification and diagnosis of inborn errors of cobalamin metabolism. These have been augmented with ES and targeted gene sequencing with gene panels to identify causal genetic variants in cobalamin-related genes. These methodologies have allowed reliable and efficient molecular diagnosis of cobalamin disorders, ${ }^{4,5}$ as well as the discovery of novel genes implicated in the pathway. ${ }^{6-9}$ However, a set of patients has accumulated over the years in which no genetic diagnosis could be identified.

In the present study, we report the results of GS and RNA sequencing in 26 patients with persistent elevated MMA, decreased fibroblast MCM function, and no diagnosis following somatic cell studies and targeted next-generation sequencing (NGS), to determine whether these analyses could identify any genetic defects that had been missed in these patients.

\section{MATERIALS AND METHODS}

\section{Patient selection and phenotyping}

Twenty-six subjects were selected from a larger cohort of 127 patients referred to the diagnostic laboratory at the Vitamin $\mathrm{B}_{12}$ Clinical Research Laboratory (Division of Medical Genetics, Department of Specialized Medicine, McGill University Health Centre [MUHC]) due to elevated levels of

\footnotetext{
${ }^{1}$ Department of Human Genetics, McGill University, Montreal, QC, Canada; ${ }^{2}$ Division of Medical Genetics, Department of Specialized Medicine, McGill University Health Centre, Montreal, QC, Canada; ${ }^{3}$ Division of Medical Biochemistry, Department of Specialized Medicine, McGill University Health Centre, Montreal, QC, Canada. Correspondence: David S. Rosenblatt (david.rosenblatt@mcgill.ca)
} 
methylmalonic acid in blood or urine, and in whom no diagnosis had been made. This project (MEDA-2000-943) was approved by the MUHC Research Ethics Board. Only results potentially related to the clinical findings of the patients were returned to the referring physicians; no incidental findings were returned. All fibroblasts had undergone testing for propionate incorporation (a measure of cellular MCM function), methyltetrahydrofolate incorporation (a measure of cellular methionine synthase function) and synthesis of cobalamin coenzyme derivatives from exogenous cyanocobalamin, ${ }^{10}$ and sequencing with an expanded version of the Baylor Miraca Genetics Laboratories Cobalamin Metabolism Panel. ${ }^{5}$ Selected patients had $\left[{ }^{14} \mathrm{C}\right]$ propionate incorporation values lower than $5.6 \mathrm{nmol} / \mathrm{mg}$ protein $/ 18 \mathrm{~h}$ (reference range: $10.8 \pm 3.7 \mathrm{nmol} / \mathrm{mg}$ protein $/ 18 \mathrm{~h}$ ). In all cases propionate incorporation was decreased compared with that of control fibroblasts assayed at the same time. A description of these patients can be found in Table 1. MMA levels in these patients were elevated compared with reference values, although not as high as in most patients diagnosed with inborn errors of cobalamin metabolism. Values for MMA in urine, when available, ranged from 5.5 to $88 \mathrm{mmol} / \mathrm{mol}$ creatinine (reference $0-3.6 \mathrm{mmol} / \mathrm{mol}$ creatinine); plasma MMA values ranged from 0.4 to $180,000 \mu \mathrm{mol} / \mathrm{L}$ (reference values $0-0.4 \mu \mathrm{mol} / \mathrm{L}$ ). When noted, blood vitamin $B_{12}$ was within the reference range; the reduced propionate incorporation values noted in cultured patient fibroblasts indicates that dietary cobalamin deficiency likely does not explain the clinically observed MMA elevations.

\section{Genomic DNA and RNA preparation}

Genomic DNA was extracted from patient fibroblasts using the FlexiGene DNA Kit (Qiagen). Concentration and purity of DNA were assessed using the BioDrop $\mu$ LITE spectrophotometer (BioDrop, UK) and the Qubit Fluorometer (Thermo Fisher). Extracts were stored at $-20^{\circ} \mathrm{C}$. For RNA sequencing studies, confluent fibroblasts were harvested, pelleted, and dissolved in $1 \mathrm{~mL}$ of Trizol Lysis Reagent (Sigma Aldrich) and immediately frozen at $-80^{\circ} \mathrm{C}$. The suspensions were used to extract total RNA using the miRNAeasy Mini Kit (Qiagen).

\section{Sequencing and CNV analysis}

Genomic DNA and complementary DNA (cDNA) were used for GS and RNA sequencing at the McGill University and Genome Quebec Innovation Center using the Illumina polymerase chain reaction (PCR)-free shotgun sequencing technology. ${ }^{11,12}$ GS was performed on $500 \mathrm{ng}$ to $1.5 \mu \mathrm{g}$ of genomic DNA using a PCR-free protocol. Libraries were sequenced on the Illumina HiSeq X platform with 151-bp paired-end reads. Results were received in uBAM file format (unaligned, raw sequencing reads). Quality control metrics were tested and showed an average of $98 \%$ of mapped reads per sample. Data analysis followed the Genome Analysis Toolkit (GATK) best practices guidelines that were developed in the Data Sciences Platform at the Broad Institute (https:// software.broadinstitute.org/gatk/best-practices/). The uBAM files were then processed through a customized data analysis pipeline to generate a filtered variant call set. Copy-number variations (CNVs) were detected using PopSV. ${ }^{13}$

\section{Variant analysis and interpretation}

Variants (single-nucleotide variants [SNVs] and indels) were filtered based on quality metrics, allele frequency, function prediction scores, and clinical relevance of variants. More specifically, for quality metrics, a variant is required to satisfy all criteria that include $62.5 \%$ or more of the samples to have called genotypes, an allele count equal to or less than 5 among all samples, greater than or equal to $10 \times$ coverage in the sample of interest, greater than or equal to 3 alternative allele reads. For allele frequency, a variant is required to satisfy all criteria, including an overall allele frequency in gnomAD less than $0.5 \%$, African allele frequency in gnomAD less than $1 \%$, European (non-Finnish) allele frequency in gnomAD less than $1 \%$, East Asian allele frequency in gnomAD less than $1 \%$, number of homozygotes in gnomAD less than 5 , and not more than $5 \%$ minor allele frequency in each population. In addition to an extensive set of function prediction scores that were added (Supplemental Data 1) a variant was required to satisfy at least one of the criteria listed in these scores to be called.

\section{Clinical interpretation of variants}

Variant interpretation was applied according to the American College of Medical Genetics and Genomics guidelines for variant prioritization. ${ }^{2}$ Specifically, when examining variants in genes potentially related to the phenotype, whether they had previously been reported as pathogenic, likely pathogenic, or a variant of unknown significance was taken into consideration. A systematic approach was applied by starting with ClinVar significance, allele frequency databases, disease association, and gene function. CNVs were manually inspected using DECIPHER.

\section{RESULTS}

We confirmed heterozygous variants of unknown significance in nine patients (Table 2) that had been previously detected by the Baylor Miraca panel. However, analysis did not identify a second pathogenic variant in any of these patients. No novel putative pathogenic variants were discovered in any known cobalamin gene, nor were biallelic variants in genes of unknown function that might represent previously unknown cobalamin metabolic genes identified. Analysis of mitochondrial DNA identified no putative pathogenic variants.

In three patients, candidate variants that could explain part of the non-cobalamin-related clinical phenotype were identified. WG2625 was found to be compound heterozygous for a nonsense variant (c.1749_1750delGGinsTT (p.Glu584*; NM_000282.3) and an intragenic 9-kb duplication (Supplementary Fig. 1) affecting exon 21 of PCCA, which encodes the a-subunit of propionyl-CoA carboxylase. These variants have not been previously reported. RNA-seq indicated reduced 


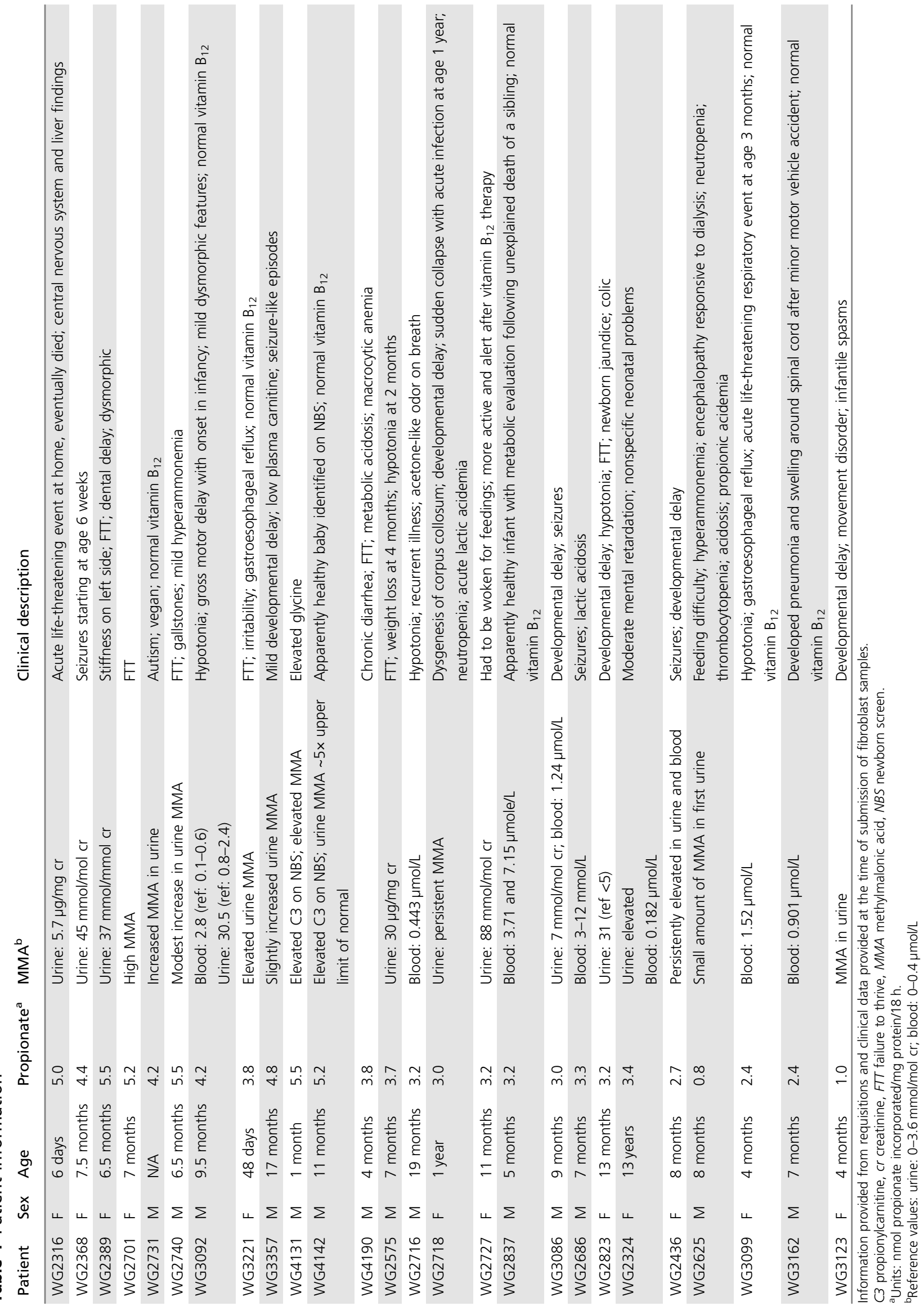


Table 2 Patients with variants previously detected by a cobalamin gene panel

\begin{tabular}{lllll} 
Patient & Sex & Gene & Nucleotide variant & Amino acid variant \\
\hline WG2436 & F & GIF & c.435_437delGAA & p.K145del \\
WG2625 & M & CUBN & c.9986C>T & p.S3329L \\
WG2701 & F & MTHFR & c.1333C>T & p.R445W \\
WG2731 & n/a & ACSF3 & c.854C>T & p.P285L \\
WG2837 & M & ACSF3 & c.1672C>T & p.R558W \\
WG3086 & M & CUBN & c.2594G>A & p.S865N \\
& & $C U B N$ & c.6469A>G & p.N2157D \\
WG3357 & M & HCFC1 & c.4442C>T & p.T1481M \\
WG4131 b & M & CD320 & c.262_264delGAG & p.E88del \\
& & $C D 320$ & c.658G>A & P.G220R \\
WG3023 & F & HCFC1 & c.4475C>T & p.P1492L \\
\hline
\end{tabular}

All detected sequence variants were heterozygous. DNA nucleotide +1 is the $A$ of the ATG translation initiation codon in the reference sequence. Reference sequences for the variants described in the table are NM_005142.2, GIF; NM_001081.3, CUBN; NM_005957.4, MTHFR; NM_174917.4, ACSF3; NM_005334.2, HCFC1; NM_016579.3, CD320; NM_000282.3， PCCA; NM_002354.2, EPCAM.

a $V$ ariant c.6469A $>\mathrm{G}$ lies outside the region of CUBN associated with cobalamin defects, and clinical phenotype does not match that of CUBN deficiency. ${ }^{4}$

${ }^{b}$ Clinical phenotype does not match that of CD320 deficiency. ${ }^{4}$

expression of the allele carrying the missense change, suggesting nonsense-mediated decay. In WG4190, GS revealed a homozygous insertion (c.499dup, p.Gln167fs) in EPCAM (NM_002354.2), which results in a frame shift leading to creation of a stop codon. which results in a frameshift leading to creation of a stop codon. Biallelic variants in this gene have previously been associated with diarrhea-5 with congenital tufting enteropathy, a rare autosomal recessive intractable diarrhea of infancy. ${ }^{14}$ Since EPCAM is only weakly expressed in fibroblasts, RNA-seq analysis was uninformative. GS and CNV analysis of WG2716 revealed a duplication at chromosome $17 \mathrm{q} 12$ encompassing approximately $1.4 \mathrm{Mb}$, spanning positions chr17: 34,815,001 to $36,250,000$ (Supplementary Fig. 2). Duplications in this region have been associated with a syndrome with variable presentation, with neurologic, behavioral, and dysmorphic features. $^{15,16}$

\section{DISCUSSION}

Somatic cell studies, including complementation analysis and gene panel analysis, are both used for diagnosis of inherited cobalamin metabolic disorders. In this study, we used GS and RNA-seq to assess the added diagnostic value of these methods in a panel of 26 patients with elevated MMA who could not be diagnosed using other technologies. In this cohort of patients, elevated MMA and low $\left[{ }^{14} \mathrm{C}\right]$ propionate incorporation suggested a possible disorder of cobalamin metabolism even in the absence of identified causal variants in any known cobalamin metabolic gene.

Propionate incorporation in these patients was less than 5.6 $\mathrm{nmol} / \mathrm{mg}$ protein $/ 18 \mathrm{~h}$, more than one standard deviation less than the mean value in fibroblasts from individuals with no inborn error of cobalamin metabolism $(10.8 \pm 3.7 \mathrm{nmol} / \mathrm{mg} /$
$18 \mathrm{~h}$ ). In 14 patients, no sequence variants of interest were found. In nine patients, heterozygous variants of unknown significance (VUS) in cobalamin metabolic genes (previously identified by the gene panel) were confirmed. In three patients, variants in genes not known to have any role in cobalamin metabolism were identified. These findings could explain some clinical findings in these patients unrelated to MMA metabolism.

Patient WG2625 was reported to have vitamin $\mathrm{B}_{12^{-}}$ responsive methylmalonic acidemia, neutropenia, thrombocytopenia, and ketotic hyperglycinemia. His first urine was reported to contain elevations of both MMA and propionic acid. Compound heterozygous sequence variants in PCCA, which encodes the $\alpha$-subunit of propionyl-CoA carboxylase, were identified in this patient. ${ }^{17}$ While neither variant identified in the present study has been previously reported, both are predicted to be deleterious. The c.1749_1750insTT variant results in a frameshift and creation of a premature stop codon; RNA-seq indicates that this variant allele is not expressed. The 9-kb duplication could not be detected by exome sequencing, demonstrating the importance of additional technologies to detect causal sequence changes such as small CNVs. Identification of biallelic PCCA variants in WG2625 suggests that propionic acidemia, rather than methylmalonic acidemia, is the correct diagnosis in this case, although the cause of the apparent elevation of MMA in the patient is unknown.

Patient WG4190 had chronic diarrhea, failure to thrive, metabolic acidosis, and macrocytic anemia. GS revealed a homozygous frameshift variant in EPCAM, which encodes the epithelial cellular adhesion molecule EpCAM. Biallelic EPCAM variants have been associated with congenital tufting enteropathy. ${ }^{14}$ This finding fits the patient's phenotype of chronic diarrhea but cannot explain the observed methylmalonic aciduria.

Patient WG2716 was reported to have hypotonia, head lag, and recurrent illnesses associated with an acetone-like odor to his breath. He also suffered from severe eczema, mild developmental delay, difficulty in gaining weight, and intermittently increased MMA in blood and urine. GS and RNA-seq identified a duplication affecting chromosome 17q12. Submicroscopic duplications of this region have been associated with a clinically variable autosomal dominant syndrome of developmental delay and other neurologic and behavioral problems as well as dysmorphology affecting the brain, kidneys, and other organs. ${ }^{15,16}$ The duplication identified in WG2716 may underlie neurologic aspects of his clinical presentation, but cannot explain the patient's elevated MMA; no gene involved in cobalamin metabolism has been localized to chromosome 17q12.

Our results show that somatic cell studies and gene panel testing are sufficient for diagnosis of inborn errors of cobalamin metabolism, since GS and RNA-seq did not result in any additional diagnoses. Elevated MMA levels in these patients remain unexplained. However, levels could be elevated for reasons not related to cobalamin metabolism, as 
occurs in patients with variants in $A C S F 3,{ }^{18}$ although this would not explain the decreased MCM function, as shown by decreased propionate incorporation. The non-cobalaminrelated findings detected in three patients could not have been detected using the gene panel, and show the added benefit of using GS to achieve a clinical diagnosis. NGS panels are now usually the first-line diagnostic approach for these disorders. Somatic cell studies including complementation analysis remain useful in cases in which clearly pathogenic sequence variants are not detected.

\section{SUPPLEMENTARY INFORMATION}

The online version of this article (https://doi.org/10.1038/s41436019-0640-9) contains supplementary material, which is available to authorized users.

\section{ACKNOWLEDGEMENTS}

We thank the patients and their families as well as the physicians who submitted patients to the laboratory for diagnosis. We also thank Jocelyne Tossa, Keo Phommarinh, and Sina Yak for laboratory assistance; Brian Gilfix and Jacek Majewski for helpful discussions; and Judith St-Onge for her expertise and guidance. We thank Calcul Québec and Compute Canada for highperformance computing resources for genomic data analysis and storage. This research was supported by Operating Grant PJT148661 from the Canadian Institutes of Health Research and is a publication of the Hess B. and Diane Finestone Laboratory in Memory of Jacob and Jenny Finestone.

\section{DISCLOSURE}

The authors declare no conflicts of interest.

Publisher's note: Springer Nature remains neutral with regard to jurisdictional claims in published maps and institutional affiliations.

\section{REFERENCES}

1. Cummings BB, Marshall JL, Tukainen T, et al. Improving genetic diagnosis in Mendelian disease with transcriptome sequencing. Sci Transl Med. 2017:9:386

2. Richards S, Aziz N, Bale S, et al. Standards and guidelines for the interpretation of sequence variants: a joint consensus recommendation of the American College of Medical Genetics and Genomics and the Association for Molecular Pathology. Genet Med. 2015;17:405-424.

3. Watkins $D$, Rosenblatt DS. Inborn errors of cobalamin absorption and metabolism. Am J Med Genet C Semin Med Genet. 2011;157:33-44.

4. Chu J, Pupavac M, Watkins $D$, et al. Next generation sequencing of patients with mut methylmalonic aciduria: Validation of somatic cell studies and identification of 16 novel mutations. Molec Genet Metab. 2016;118:264-271.

5. Pupavac M, Tian X, Chu J, et al. Added value of next generation gene panel analysis for patients with elevated methylmalonic acid and no clinical diagnosis following functional studies of vitamin $\mathrm{B}_{12}$ metabolism. Mol Genet Metab. 2016;117:363-368.

6. Coelho D, Kim JC, Miousse IR, et al. Mutations in $A B C D 4$ cause a new inborn error of vitamin $B_{12}$ metabolism. Nat Genet. 2012;44: 1152-1155.

7. Yu HC, Sloan JL, Scharer G, et al. An X-linked cobalamin disorder caused by mutations in transcriptional coregulator HCFC1. Am J Hum Genet. 2013;93:506-514.

8. Pupavac $M$, Watkins $D$, Petrella $F$, et al. Inborn error of cobalamin metabolism associated with the intracellular accumulation of transcobalamin-bound cobalamin and mutations in ZNF143, which codes for a transcriptional activator. Hum Mutat. 2016;37:976-982.

9. Quintana AM, Yu HC, Brebner A, et al. Mutations in THAP11 cause an inborn error of cobalamin metabolism and developmental abnormalities. Hum Mol Genet. 2017;26:2838-2849.

10. Watkins D, Matiaszuk N, Rosenblatt DS. Complementation studies in the cbla class of inborn error of cobalamin metabolism: evidence for interallelic complementation and for a new complementation class $(\mathrm{cb} / \mathrm{H})$. J Med Genet. 2000;37:510-513.

11. Witkowski L, Carrot-Zhang J, Albrecht S, et al. Germline and somatic SMARCA4 mutations characterize small cell carcinoma of the ovary, hypercalcemic type. Nat Genet. 2014;46:438-445.

12. Guéant JL, Chéry C, Oussalah A, et al. A PRDX1 mutant allele causes a MMACHC secondary epimutation in cblC patients. Nat Commun. 2018;9:67.

13. Monlong J, Girard SL, Meloche C, et al. Global characterization of copy number variants in epilepsy patients from whole genome sequencing. PLoS Genet. 2018;14:e1007285.

14. Sivagnanam $M$, Mueller JL, Lee $H$, et al. Further evidence for EpCAM as the gene for congenital tufting enteropathy. Am J Med Genet A. 2008;152A:222-224.

15. Bierhals T, Maddakuri SB, Kutsche K, Girisha KM. Expanding the phenotype associated with 17q12 duplication: case report and review of the literature. Am J Med Genet A. 2013;161A:352-359.

16. Kamath A, Linden SC, Evans FM, et al. Chromosome 17q12 duplications: further delineation of the range of psychiatric and clinical phenotypes. Am J Med Genet B. 2018;177B:520-528.

17. Rivera-Barahona A, Navarrete R, Garcia-Rodriguez R, et al. Identification of 34 novel mutations in propionic acidemia: functional characterization of missense variants and phenotype associations. Mol Genet Metab. 2018; $125: 266-275$

18. Levtova A, Waters PJ, Buhas D, et al. Combined malonic and methylmalonic aciduria due to ACSF3 mutations: benign clinical course in an unselected cohort. J Inherit Metab Dis. 2019;42:107-116. 